\title{
GeospatialVR: A Web-based Virtual Reality Framework for Collaborative Environmental Simulations
}

Yusuf Sermet ${ }^{1}$, Ibrahim Demir ${ }^{2}$

${ }^{1}$ IIHR - Hydroscience \& Engineering, University of lowa, lowa City, IA, USA

${ }^{2}$ Civil and Environmental Engineering, University of lowa, lowa City, IA, USA

\section{Contact}

Yusuf Sermet

msermet@uiowa.edu

\section{Highlights}

- Development of an open-source virtual reality framework to dynamically simulate any location in the world.

- Development of a JavaScript API to serve and manage VR scenes on any web platform.

- Support for real-time multiuser interaction with personalized avatars.

- Demonstration of the framework for flooding, wildfire, transportation, and active shooter use cases.

This manuscript is an EarthArXiv preprint and has been submitted for possible publication in a peerreviewed journal. Please note that the manuscript has yet to be formally accepted for publication. Subsequent versions of this manuscript may have slightly different content. If accepted, the final version of this manuscript will be available via the 'Peer-reviewed Publication DOI' link on the righthand side of this webpage. Please feel free to contact the corresponding author for feedback. 


\begin{abstract}
This research introduces GeospatialVR, an open-source collaborative virtual reality framework to dynamically create 3D real-world environments that can be served on any web platform and accessed via desktop and mobile devices and virtual reality headsets. The framework can generate realistic simulations of desired locations entailing the terrain, elevation model, infrastructures, dynamic visualizations (e.g. water and fire simulation), and information layers (e.g. disaster damages and extent, sensor readings, occupancy, traffic, weather). These layers enable in-situ visualization of useful data to aid public, scientists, officials, and decision-makers in acquiring a bird's eye view of the current, historical, or forecasted condition of a community. The framework incorporates multiuser support for remote virtual collaboration. GeospatialVR's purpose is to augment cyberinfrastructure systems with immersive geospatial capabilities to assist development of next-generation information and decision support systems powered by virtual reality. Finally, several case studies have been developed for flooding, wildfire, transportation, and active shooter response.
\end{abstract}

\title{
Keywords
}

virtual reality; geospatial visualization; disaster management; decision-support systems; web-based interaction

\section{Software availability}

Name

Geospatial VR

Developers

Yusuf Sermet (msermet@uiowa.edu)

Contact information

300 S. Riverside Dr., lowa City, IA 52246, USA

Software required Internet browser

Program language HTML5, JavaScript (client side), C\#, Unity (core engine), Node.js (multiuser server)

Availability and cost The framework is open-source and free to use. The repository can be accessed on GitHub.

Code repository https://github.com/uihilab/GeospatialVR

\section{Introduction}

Huge amounts of spatial data are being generated at an increasingly accelerating rate due to the advancements in remote sensing and availability of internet-enabled sensors (Kruger et al., 2016; 
Sermet et al., 2020b). Current approaches to turn the massive stream of data into actionable knowledge involves the development of domain-specific information systems on the web (Zhang et al., 2019; Wang et al., 2020). Though they prove to be useful in informing the public and aid decision-makers and stakeholders of a domain (e.g. disasters, transportation, urban planning, agriculture) (Demir et al., 2018), they still suffer from the complexity and variance of available parameters which presents a challenge for informed decision-making pertaining to a task in its geospatial context in the real-world (Salas et al., 2020; Xu et al., 2020). As the prevalence of smart devices (e.g. laptops, mobile phones, immersive headsets) equipped with graphical processing units (GPU) capable of high-quality 3D rendering increase (Turner et al., 2016), immersive visualization and 3D modelling provides a great potential to constitute the next-generation of map-based information systems where real-world location can be recreated with in-situ spatiotemporal data displays and simulated event dynamics (Chen and Lin, 2018; Wang et al., 2019). Such immersive systems pave the way for collaborative decisionmaking by allowing multiple stakeholders to interact with the same virtual environment (Chen et al., 2020).

In pursuit of this immersive system vision, there have been several studies on utilizing 3D visualizations as a means of education, training, and decision-making in fields where spatial data is involved (Lv et al., 2016; Han et al., 2018; El Sayad et al., 2019). As an early example, SIEVE offers a collaborative 3D modelling framework where specialists can use GIS data to construct 3D models that can be downloaded and viewed via a desktop application (Stock et al., 2008). More recent research shows how $360^{\circ}$ photos can be utilized to create the perception of a virtual environment where users can place and examine textual 2D information layer overlays (e.g. markers), which may present use cases for tour guides (Masrur et al., 2017; Wallgrün et al., 2019). However, enabling immersive spatial data visualization, exploration, and analysis as a geographical information system requires dynamic construction of roamable real-world VR environments allowing physical interaction (Abdalla and Esmail, 2019). Handling and analyzing large scale datasets on the web for immersive applications can benefit from optimized data models (Sit et al., 2019), client-side processing (Demir and Szczepanek; 2017) and voluntary distributed computing approaches (Agliamzanov et al., 2020). To discover the simulation opportunities that are brought by immersive systems for decision-making purposes, several studies examine how 3D analytics and realistic simulations of environmental events can be incorporated into virtual experiences (Simpson et al., 2016; Winkler et al., 2018; Kolb et al., 2019; Cornel et al., 2019). 
More specifically, in the area of disaster preparedness and mitigation, previous work shows individual efforts for 3D visualization and real-world scene creation for preprocessed locations where external data resources can be integrated (Bouloukakis et al., 2018; Koutitas et al., 2019; Fujimi and Fujimura, 2020). Macchione et al. (2019) proposed a workflow to represent realistic two-dimensional hydraulic and flood simulations on a preprocessed 3D virtual environment within Blender software, and demonstrate the functionality for Cosenza, Italy. For firefighter training, Nahavandi et al. (2019) introduced a VR system that features particle modelling to employ water dispersion as well as its interaction with fire and smoke objects. Feng et al. (2020) presented a virtual reality serious game for earthquake emergency training and conducted a case study that reports that the participants' understanding of earthquake preparedness and evacuation were increased post-training. Flood Action VR (Sermet and Demir, 2019a) and HoloFlood are virtual and augmented reality applications that utilize historical, current, or forecasted weather, disaster, and geographic data to construct a 3D virtual model of a city with a flood simulation where stakeholders can examine flood extent and damage estimations (Sermet and Demir, 2020).

The literature displays a significant gap in and need for collaborative and generalized immersive platforms for environmental decision-making (Havenith et al., 2019). Such platforms require realization with a device-independent solution that can be accessed directly from the web and that can be powered on the fly for any location. Furthermore, conventional procedures for creating immersive experiences are costly in terms of the effort, time, hardware resources, and technical merit it requires, which presents difficulties in adopting these technologies and taking full advantage of their benefits for training, education, and decision-making (Leder et al., 2019; U.S. Army, 2020). Finally, widespread adoption of such dynamic interfaces and next-generation interactions are dependent upon free and open-source solutions (Mind Commerce, 2020).

This paper introduces GeospatialVR, an open-source collaborative virtual reality framework to dynamically create 3D real-world environments that can be served on any web platform and accessed via desktop and mobile devices as well as virtual and augmented reality headsets. The framework is capable of generating realistic simulations of desired locations entailing the terrain, elevation model, structures (e.g. buildings, roads, bridges), dynamic visualizations (e.g. water and fire simulation), and information layers (e.g. disaster damages and extent, sensor readings, surveillance data, occupancy, traffic, weather). These layers serve the purpose of effective and in-situ visualization of useful data to 
aid public, scientists, officials, and decision-makers in acquiring a bird's eye view of the current, historical, or forecasted condition of a community. The framework incorporates multiuser support to allow different stakeholders to remotely work on the same VR environment and observe other user's actions and 3D positions via avatars in real-time. The purpose of GeospatialVR is to augment web-based cyberinfrastructure systems with immersive geospatial capabilities to assist development of nextgeneration information systems (IS) and decision support systems (DSS) powered by virtual and augmented reality technologies.

The main contributions of this research can be summarized as follows. The presented framework is designed and implemented as a generalized system that can be adopted anywhere in the world by simply providing the latitude and longitude of the desired location. All 3D modeling and generation processes are done on-the-fly utilizing client-side hardware resources without requiring preprocessing. To aid the adopters of the system in customizing the application for their use cases, a JavaScript API has been developed for users to provide their geospatial data layers in the form of JSON file, which automatically is mapped and visualized on the 3D map. The multiuser support provides a collaborative VR environment to enable users to work on the same interactive simulation, and thus, presenting a potential to be utilized as a virtual incident command center or a meeting room. The unique design of GeospatialVR's system architecture and software leads to its usage on any modern browser as well as access to a fully immersive VR environment via virtual and augmented reality headsets (e.g. HTC Vive, Oculus, Windows MR headsets). Finally, the generalized and well-documented design of the presented framework encourages further research and extensions in accordance with the best practices put forth for the software-based geoscience research (Gil et al., 2016).

The remainder of this article is organized as follows. Section 2 presents the system design and components, and describes the methodology. Section 3 focuses on the implementation of the presented framework in multiple use cases to demonstrate its generalized nature and applicability. Section 4 discusses future work and provides conclusions.

\section{Methods}

The GeospatialVR framework consist of three main components; the core engine, multiuser management server, and web applications. The core engine is developed with C\# in Unity3D game engine and exported as a JavaScript library, which entails the core functionalities of the system such as 
data retrieval and dynamic scene generation, compatibility support for web rendering and VR, communication and management of the scene by client-side applications, and scene synchronization for multiuser support. Web applications can be developed and augmented utilizing this JS library, which is capable of communicating with an external server for user management. The server has been developed with Node.js to serve as a centralized multiuser management module to synchronize the scene between different clients. Figure 1 summarizes the framework components and their relationships and communication.

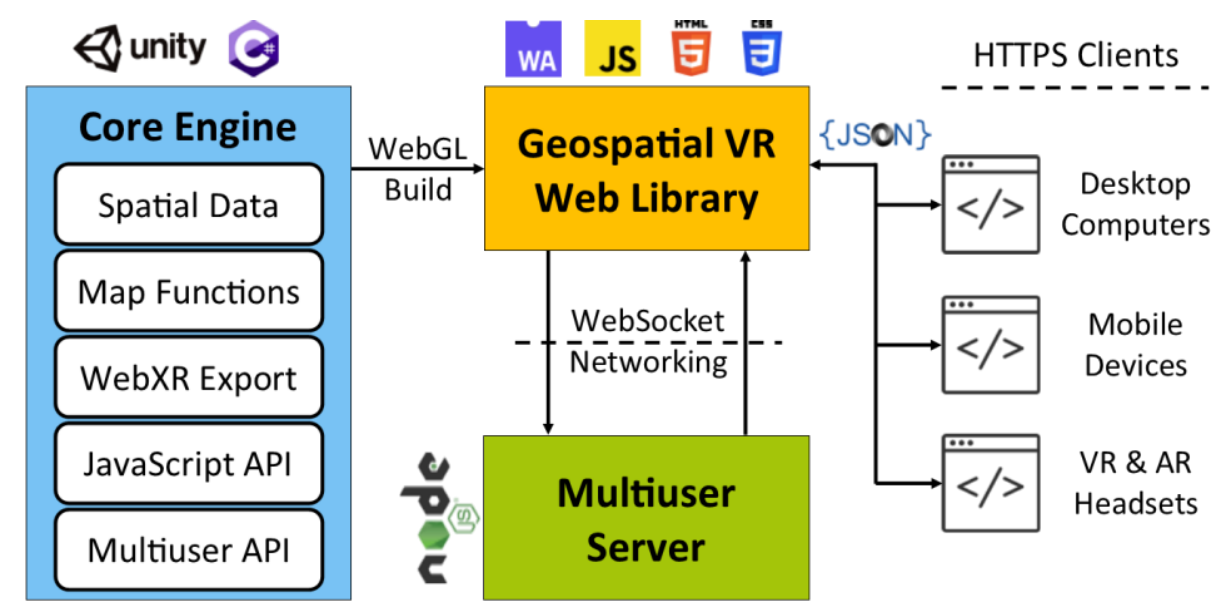

Figure 1. System architecture of GeospatialVR framework.

\subsection{Core Engine}

\subsubsection{Spatial Data and Map Functions}

The framework is powered by various data resources to create realistic representations of real-world locations on demand. Within the context of this research, Mapbox software development kit (SDK) has been selected to retrieve world-wide terrain, street, elevation, texture, and structural data and import map functions due to its customizable nature and being free-to-use. Modifications have been made to the SDK to incorporate a comprehensive and modern vector tiling system (Mapbox Streets v8) despite its experimental nature and current incompatibility with certain features of the map services such as defining points of interests. The vector tiling system provides a structured and labeled map streaming service powered by various data sources including Open Street Maps, Microsoft Open Maps, and Wikidata. The labeled nature of map tiles allow the customization of terrain features (e.g. water, buildings, roads) with animated materials and object colliders for the creation of an interactable and immersive experience. For example, virtual reality-compatible water shaders have been used to create 
polygons overlaid onto water bodies to simulate flow that introduce the option to be affected by scene conditions such as wind and weather. The integrated map services allow dynamic manipulation of the generated 3D map object based on its location, extent, map styles, terrain elevation scale, as well as the functionality for conversion between real-world and game scene coordinates.

Complementing the core map functionalities, additional features have been developed and integrated into the core engine to add data layers and geospatial visualizations. Custom prefabs have been defined with identifiers and slots to allow dynamic instantiation of points of interests and data labels attached to the corresponding game position for given latitude and longitude. Though the possibilities are limitless, examples to use cases of this functionality include displaying in-situ sensor readings (e.g. water depth, rain gauge, soil moisture, disaster warnings), on-site people (e.g. personnel, officers, first responders) along with associated data (e.g. name, pulse, oxygen saturation), and forecasts and risk assessments for specific locations and buildings (e.g. potential disaster damage, vulnerable population, occupancy for a time-of-the-day). In addition to text-based 3D data points, some parameters can be used to support dynamic visualizations and animations for an immersive experience as well as the opportunity to observe the interaction of natural events. Examples to such visualizations can include the conversion of water depth data to simulate floods, heat sensor data for simulating wildfires to observe its path and progression, traffic data to visualize congestion, and weather data to simulate wind, fog, and precipitation.

\subsubsection{D Visualization and Virtual Reality on the Web}

One of the goals of the framework is to be compatible with different types of devices to ensure maximum availability and prevent dependency to any specific platform. However, this is a major challenge as many virtual reality devices have different capabilities, interaction methods, and requires special development processes due to their varying software stacks. VR devices are not yet commonplace due to still being a maturing technology (Lv, 2019) and not being standardized. Many existing information systems and decision support systems are served as one-stop web applications. As a solution, this project benefits from the modern web specifications of WebXR and WebVR, to create a web-based virtual reality-enabled 3D application. This allows the system work like a conventional web application accessed via desktop and mobile browsers while allowing the transition to a fully immersive experience on VR devices (e.g. HTC Vive, Oculus Rift and Quest, HP Reverb). Since WebXR is an experimental technology with continuously changing standards (Butcher et al., 2020), an open-source 
asset (i.e. Unity Web Exporter by Mozilla) is utilized to export the core engine into a JS library to ensure a consistent and up to date application generation process for WebXR.

\subsubsection{Client Communication API}

Software development in Unity with C\# is relatively more expensive in terms of the effort, time, hardware resources, and technical merit it requires in comparison to basic web development with JavaScript. More so, it is not feasible to be dependent to a compiled development environment for an application that is designed to be generalized and easy-to-use by the associates of any domain. Thus, a communication API has been implemented to allow the adopters of the compiled JavaScript library of the GeospatialVR to trigger events on, pass data to, and interact with the 3D scene on the web. Serializable classes have been designed as a general template to pass data in the JSON format that include details such as the action type (e.g. add POI, change map location, generate flood) and action parameters (e.g. geolocation, simulation data, POI label content) with minimal configurations of the use case to avoid overfitting to the needs of any certain domain and application area.

\subsubsection{Multiuser Interaction API}

An important aspect of the presented framework is to serve as a collaborative platform where different stakeholders can observe and interact with the same 3D simulation environment. To achieve this goal, there are two aspects requiring attention: representing other users with avatars and names to create the feeling of being in the same room, and synchronizing the positions and rotations of other users along with any actions taken by them. The Multiuser API employs a networking module, that is powered by WebSocket protocol (Wang et al., 2013), and communicates with an external server, which is described in Section 2.2. Each user is assigned a unique 128-bit integer value as identification (ID) at scene initialization. The core engine sends all the relevant user-specific parameters to a centralized server whenever there is a new action, or a predetermined time has passed to keep the user active on other users' environments. The API periodically polls broadcasts that contain structured information regarding simultaneous users in the same room. User ID is used to determine new participants for whom new game objects are created with provided parameters, such as location, rotation, profile (e.g. name), and actions. For existing participants, these parameters are updated. More challenging part is to synchronize any actions taken (e.g. enabling information panels, triggering event dynamics) or data uploaded by any of the users with others as they all have their own instance of the GeospatialVR system. However, the system already incorporates a Client Communication API as the formal pipeline of passing data and manipulating the model. Since the functions of the proposed system is deterministic, meaning 
that they always result in the same output given the same input, the Multiuser API simply retrieves the exact same command other user performed on their machines with identical parameters, and executes it to get the same visual and functional result on the local device. Though this mechanism presents a potential infinite loop for different users to recursively trigger the same actions, this is easily avoided by method overloading to supply a special flag indicating that a function is executed in purpose of synchronizing an action taken by an external remote participant.

\subsection{Multiuser Management Server}

An externally hosted Node.js application, that is deployed on a cloud platform (i.e. Heroku) for the use cases, is used to develop and implement a WebSocket server that acts as the main hub for managing multiple users for any web application utilizing the GeospatialVR framework, being compatible with the networking protocols of the core engine in the .NET environment. A two-way communication mechanism is defined to 1) retrieve the current status of a client application with a unique identifier and save it and 2) to periodically broadcast the most recent state of every other client application to each client. To avoid duplicate messages, status updates are cleared after each broadcast to clients, where the updates are taken into a queue for sequential execution. Identifying disconnected clients was a challenge given that web applications can be abruptly exited by simply closing a browser tab, thus, inherently not allowing the broadcast of disconnection. To resolve this issue, timestamps are recorded to keep track of received signals for active connections. When a nonresponsive client is identified, it is then broadcasted to remaining clients to update the 3D scene.

\subsection{Client Web Applications}

The GeospatialVR framework can be incorporated into any existing web platform by simply importing the JavaScript library which is generated as an export of the core engine. The library provides the initialization script to assign the 3D model to a DOM container element which can be styled and positioned per the developer's requirements. The web application communicates with and manages the 3D environment through its JavaScript API with data structured as JSON. The container element comes with basic user interfaces for scaling the 3D environment for the entire browser screen as well as a button to activate the virtual reality mode if the device used to access the website supports it. The web application is required to provide a valid certificate as the users will need to connect to the website with a secure context (e.g. https). A boilerplate web application is provided as part of the shared open-source repository to demonstrate how to import and adopt the presented framework. 


\section{Results}

Several use cases have been developed to showcase the presented framework's capabilities and its generalized nature allowing adoption by any domain where geospatial data is involved. Test data have been retrieved from federal and state organizations within the United States (US) (e.g. US Geological Survey, US National Institute of Standards and Technology, Department of Motor Vehicles, lowa Flood Center) that deal with flooding, wildfire, transportation, and active shooter situations and have established data formats, which our use cases utilize.

\subsection{Flooding Case Study}

The increased attention to matters such as climate change and sustainability (Visser et al., 2019), in combination with the advancements in sensor technologies and artificial intelligence, affordable and dense networks of internet of things (IOT) devices specialized on environmental monitoring are providing high-velocity and accurate streams of useful data (Jadidoleslam et al., 2019). Making use of this massive data for context-informed and on-site analysis with realistic visualizations and event dynamics exceeds the scope of conventional web platforms and is in need of intuitive and interactive simulation environments (Carson et al., 2018; Sermet et al., 2020a). Thus, flood management serve as a great example of how the presented framework can be valuable for public, decision-makers, and scientists on operational and research level. GeospatialVR is capable of effectively visualizing historical, current, and forecasted flood scenarios and offer realistic context for decision-making. Potential flood damages in terms of structural and content value can be estimated based on the generated flood condition and the building data which can be acquired and curated from datasets (e.g. HAZUS) or taxassessor data (Yildirim and Demir, 2019). In accordance with common practices of flood preparedness and response (Weber et al., 2018), relevant data layers can be displayed on selected locations and can be powered from static sources as well as real-time sensor streams. Figure 2 presents an urban scene where a river flood is simulated where the animated flood waters overflow from the river until it reaches to high-elevation sections of the terrain. Several sensors and gauges are incorporated to inform the stakeholders on water depth (ft), discharge $\left(\mathrm{ft}^{3} / \mathrm{s}\right)$, rain accumulation (in/hr), groundwater temperature $\left({ }^{\circ} \mathrm{F}\right)$, soil moisture at maximum probe depth (volumetric water content), wind direction and speed, and estimations for structural and content damages for buildings that are under the effect of a simulated flood scenario. Figure 2 also shows the avatar of another user who is simultaneously working on the same virtual environment. 


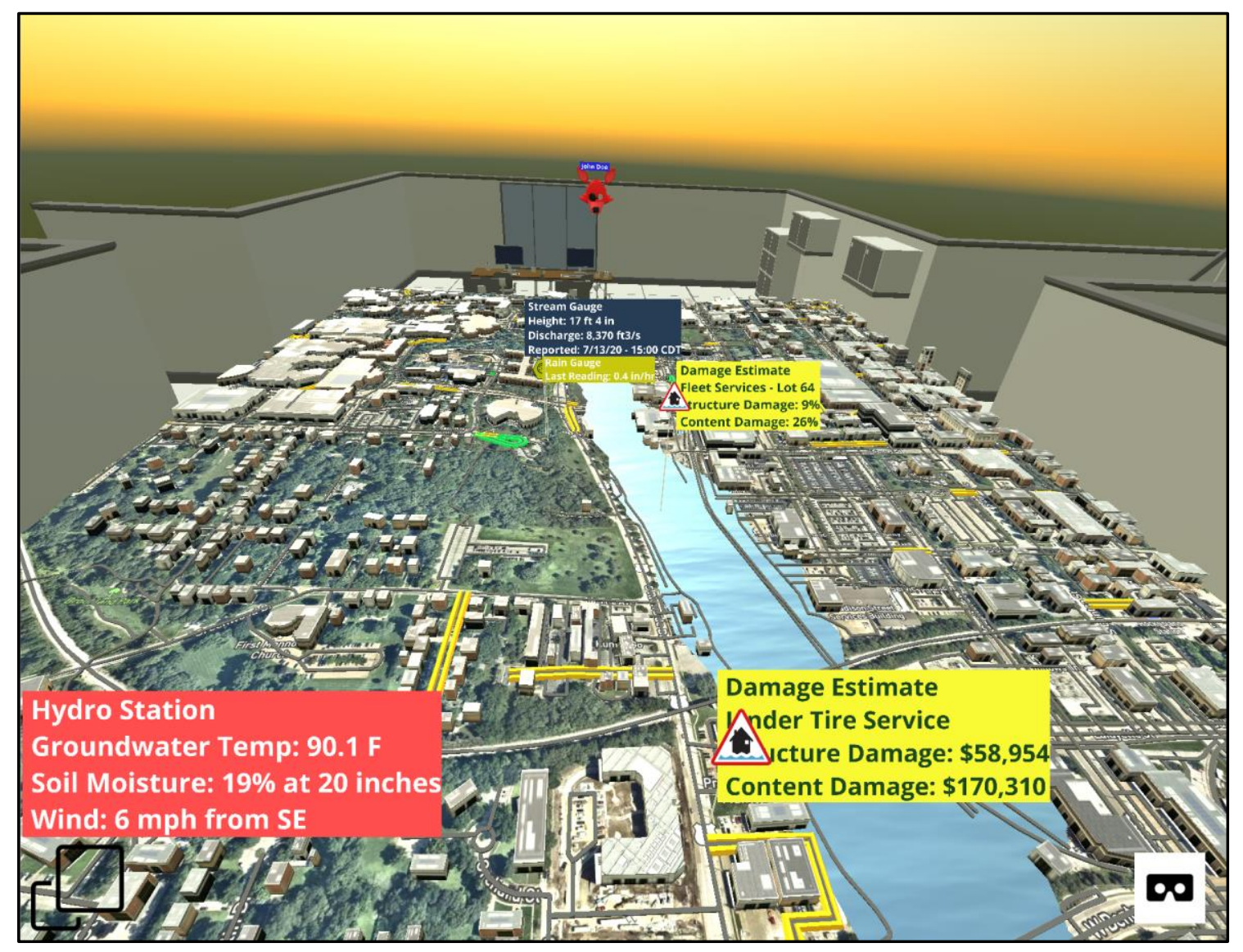

Figure 2. A case study for flood management use case for lowa City, IA, showing a flood animation and relevant data layers (i.e. stream gauges, rain gauges, hydro stations for groundwater and soil moisture data, estimated flood damages for current or forecasted flood scenarios, and traffic congestion).

\subsection{Wildfire Case Study}

Due to their wide spread and devastating effects with little opportunity for realistic drills (Labossière and McGee, 2017), virtual reality have been proved to be a beneficial tool in serving as an effective simulation environment allowing informed decision-making and observe the trends and sources of fire spread (Bogdos and Manolakos, 2013). Similar to flooding case, the proposed framework can be useful as an incident command center tool for identifying the areas and buildings at risk while evaluating the vulnerable population and economic damage, which can aid the decision makers in prioritizing rescue efforts. Raw data feeds from sensors like thermal detectors and air pollution and smoke measurement instruments can be formed to infer and visualize the fire's location on the 3D map along with its progression. 


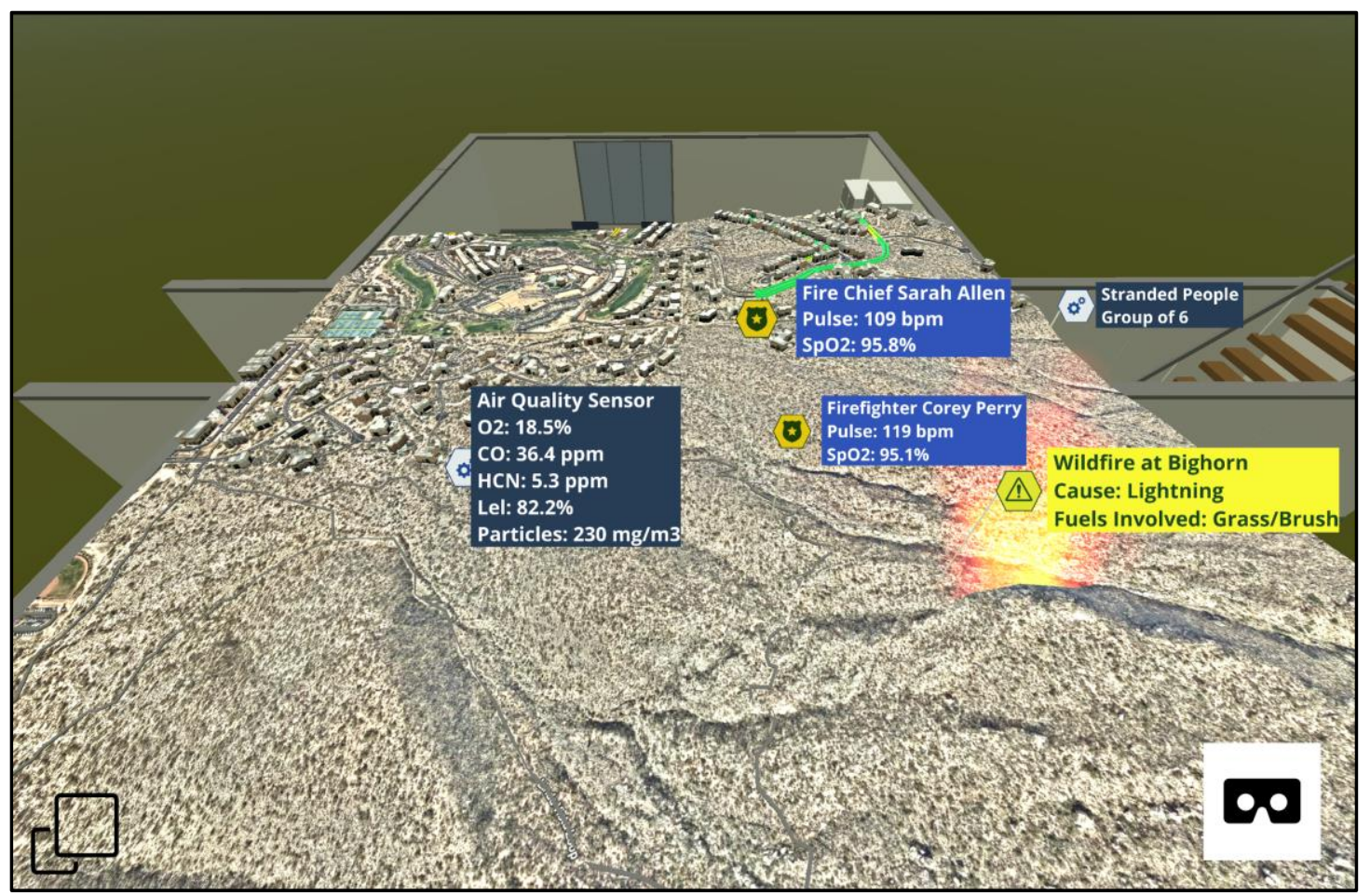

Figure 3. A case study for wildfire use case for Tucson, $A Z$, showing a fire animation and relevant data layers (i.e. characteristics and center of the wildfire, stranded groups of people detected by drones or emergency calls, air quality and gas measurements in the area, first responder vitals, and traffic congestion).

As opposed to conventional information systems, observing the real-time, historical, or forecasted condition of a wildfire in combination with optional data layers and capabilities in 3D environment (e.g. traffic-informed navigation, wind speed and direction to infer the fire growth path) provides a clear picture of the extreme event for spatially-aware decisions by taking the terrain conditions and vegetation into account. Figure 3 shows an example scenario for wildfire response. The values from the wind sensors are used to create a virtual wind object to allow further applications on fire spread.

\subsection{Transportation Case Study}

Traffic management and transportation-related accident and hazard monitoring is a prominent application area for virtual reality-powered solutions (Li et al., 2016). Thanks to the comprehensive and labeled vector tile system utilized in this framework, the roads can be augmented with realistic textures and rigid and dynamic vehicle objects based on the current traffic conditions, which can be taken into account for context-aware navigation. Point data layers can be added onto the 3D model to mark 
significant events and useful information, which include traffic accidents accompanied with relevant information such as vehicle occupants, injuries, and likely fatalities, and pertinent sensor readings for voltage levels in the railways and radiation levels that may be caused by accidents involving the transportation of radioactive materials by trucks or railways (Xie et al., 2011; CDC, 2018).

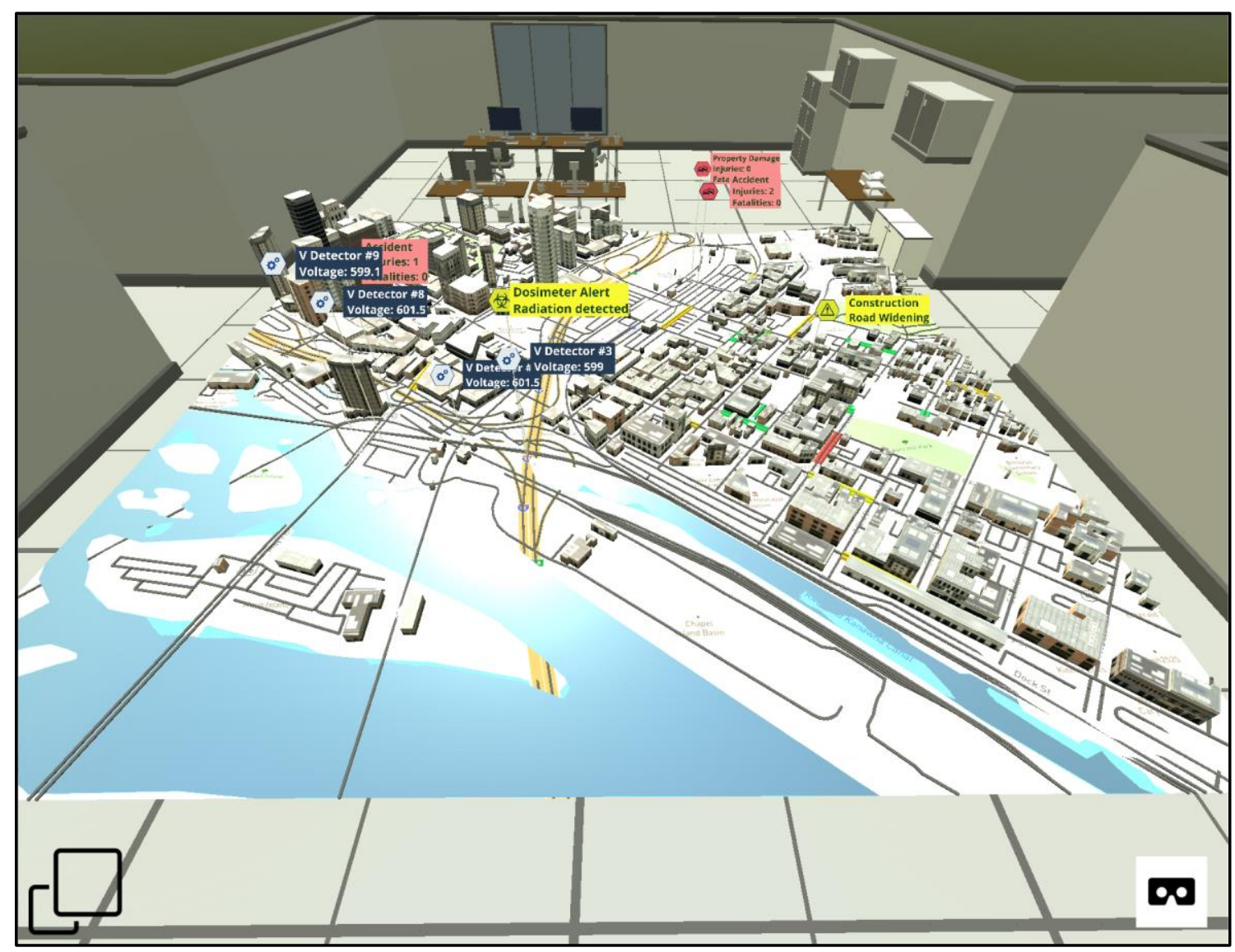

Figure 4. A case study for transportation use case for Richmond, VA, showing relevant data layers (i.e. voltage detectors, radiation spills, constructions, accident reports, and traffic congestion).

Similar to wildfire use case, air quality sensors can also be useful to monitor smoke in order to identify and locate potential accidents. Figure 4 shows an example VR scene for transportation use case. Another application area for VR in transportation is the psychological analysis for driver behavior and training by immersing subjects into realistic VR environments (Sun et al., 2019). Though this application is out of the scope of GeospatialVR's current version, the core engine can easily be scaled and modified for such use cases in the future. 


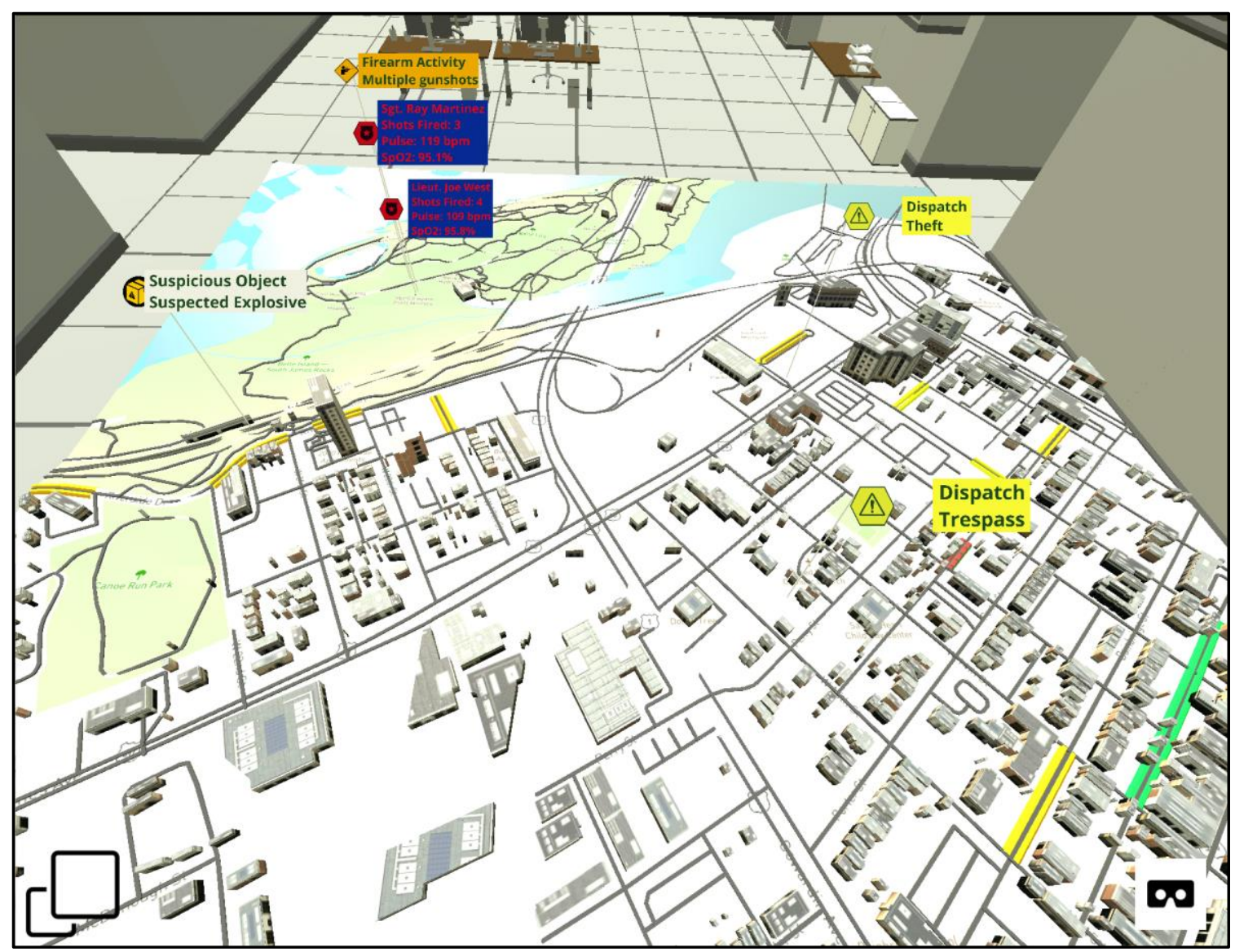

Figure 5. A case study for law enforcement, showing relevant data layers (i.e. detected gunshots, responding officers and their vitals, objects of interest, calls for dispatch, and traffic congestion).

\subsection{Active Shooter Case Study}

Active shooter incidents are posing an increasingly bigger threat in the United States (Lankford, 2019). Mass shooting can be defined as a single shooting spree where four or more people are shot (O'Rourke, 2017). There has been a total of 434 active shooter incidents that occurred in the US in 2019 alone (Wikipedia, 2020). A key to effectively and safely deal with active shooter cases lie on assuring reliable data and actionable information flow to law enforcement in real-time (Blair et al., 2013). Incident commanders at the center has significantly more information and resources at their disposal that may benefit the first responder on-site (Zhu et al., 2019). The presented VR framework can be used as an integrated decision support system to monitor an active incident, as the events progress due to the fact that shootings take place rather quickly and in movement, or to serve as a training and drill tool for preparedness. Based on various audio feeds of microphones located on officers or Internet of Things devices in the area, the location of the perpetrator can be estimated and located on the 3D model based 
on the triangulation of gunshot sounds. Incident commanders at the center can monitor the first responding law enforcement members as a live feed with information such as their vitals (i.e. pulse, oxygen saturation), weapon-drawn status, and the number of shots fired. Occupancy data for the time of the day can be displayed considering the school class schedules. The doorways and entrances of a building can be highlighted with status (i.e. locked, unlocked). Finally, suspicious objects, that may be determined by on-site cameras, drones or eyewitness reports, can be visualized on the map as a POI. Figure 5 shows an example use case powered by a federal test dataset for Richmond, Virginia.

\section{Conclusions and Future Work}

This paper presents GeospatialVR, a generalized and open-source web framework to incorporate interactive real-world environments for 3D visualizations on the web as well as immersive experiences via virtual reality headsets. The framework is capable of generating 3D models (e.g. terrain, elevation, textures, buildings, roads) of selected locations in real-time with animated visualizations (e.g. water flow, fire, traffic), dynamically adding interactive information layers for in-situ monitoring, simulating natural events (e.g. flood) based on historical, current, or forecasted conditions, and supporting multiple stakeholders to collaborate on the same virtual environment with its Multiuser API to enable collaborative decision-making. The presented framework is applicable onto any domain that deals with spatiotemporal data and is designed to support the next generation information and decision support systems with geospatial components with immersive technologies.

The study provides brief use cases to demonstrate how the presented framework can be adopted by different domains and encourages further advancements for numerous potential application areas. The modular structure of the library enables safe and simple integration of interactive and collaborative virtual environments into existing web platforms without posing a design or security complication. New event dynamics can be integrated to simulate realistic conditions and observe the interaction between natural phenomena, such as object (e.g. vehicles) movements during floods and tracking the burning process of forests and trees during wildfires (Pimont et al., 2016). In regards to multiuser immersion, the advancements in volumetric model generation out of conventional 2D pictures along with novel compression methods allowing fast-stream of 3D data (Bell and Zhang, 2019), the pictures of users can be used to create life-like avatars allowing the stakeholders to see their peers as they interact with the VR environment. Finally, voice-enabled intelligent assistants (Sermet and Demir, 2018) with domain ontologies (Sermet and Demir, 2019b) can be integrated into web platforms to retrieve actionable 
knowledge that can be helpful during the decision-making as well as for the hands-free control of the VR environment for model manipulation and overlaying data layers.

\section{ACKNOWLEDGEMENT}

This project is based upon work supported by the lowa Flood Center and the University of lowa.

\section{DISCLAIMER}

Mention of any brand name in no way implies recommendation of a particular manufacturer or product.

\section{REFERENCES}

1. Abdalla, R., \& Esmail, M. (2019). Immersive Environments for Disaster and Emergency Management. In WebGIS for Disaster Management and Emergency Response (pp. 73-78). Springer, Cham.

2. Agliamzanov, R., Sit, M. and Demir, I., 2020. Hydrology@ Home: a distributed volunteer computing framework for hydrological research and applications. Journal of Hydroinformatics, 22(2), pp.235248.

3. Bell, T., \& Zhang, S. (2019). Holo Reality: Real-time low-bandwidth 3D range video communications on consumer mobile devices with application to augmented reality. Electronic Imaging, 2019(16), 71.

4. Blair, J. P., Nichols, T., Burns, D., \& Curnutt, J. R. (2013). Active shooter events and response. CRC Press.

5. Bogdos, N., \& Manolakos, E. S. (2013). A tool for simulation and geo-animation of wildfires with fuel editing and hotspot monitoring capabilities. Environmental modelling \& software, 46, 182-195.

6. Bouloukakis, M., Partarakis, N., Drossis, I., Kalaitzakis, M., \& Stephanidis, C. (2019). Virtual Reality for Smart City Visualization and Monitoring. In Mediterranean Cities and Island Communities (pp. 1-18). Springer, Cham.

7. Butcher, P. W. S., John, N. W., \& Ritsos, P. D. (2020). VRIA: A Web-based Framework for Creating Immersive Analytics Experiences. IEEE Transactions on Visualization and Computer Graphics.

8. Carson, A., Windsor, M., Hill, H., Haigh, T., Wall, N., Smith, J., Olsen, R., Bathke, D., Demir, I. and Muste, M., 2018. Serious gaming for participatory planning of multi-hazard mitigation. International journal of river basin management, 16(3), pp.379-391.

9. Centers for Disease Control and Prevention (CDC). (2018, April 4). Types of Radiation Emergencies. Retrieved on July 12, 2020, from https://www.cdc.gov/nceh/radiation/emergencies/moretypes.htm 
10. Chen, M., \& Lin, H. (2018). Virtual geographic environments (VGEs): originating from or beyond virtual reality $(\mathrm{VR})$ ?.

11. Chen, M., Voinov, A., Ames, D. P., Kettner, A. J., Goodall, J. L., Jakeman, A. J., Barton, M., Harpham, Q., Cuddy, S.M., DeLuca, C., Yue, S. (2020). Position paper: Open web-distributed integrated geographic modelling and simulation to enable broader participation and applications. Earth-Science Reviews, 103223.

12. Cornel, D., Buttinger-Kreuzhuber, A., Konev, A., Horváth, Z., Wimmer, M., Heidrich, R., \& Waser, J. (2019, June). Interactive Visualization of Flood and Heavy Rain Simulations. In Computer Graphics Forum (Vol. 38, No. 3, pp. 25-39).

13. Demir, I. and Szczepanek, R., 2017. Optimization of river network representation data models for web-based systems. Earth and Space Science, 4(6), pp.336-347.

14. Demir, I., Yildirim, E., Sermet, Y. and Sit, M.A., 2018. FLOODSS: lowa flood information system as a generalized flood cyberinfrastructure. International journal of river basin management, 16(3), pp.393-400.

15. El Sayad, Z. T., \& Ayad, H. M. (2019). VRGIS as assistance tool for urban decision making: RafahGaza-Palestine. Alexandria Engineering Journal, 58(1), 367-375.

16. Feng, Z., González, V. A., Amor, R., Spearpoint, M., Thomas, J., Sacks, R., ... \& Cabrera-Guerrero, G. (2020). An immersive virtual reality serious game to enhance earthquake behavioral responses and post-earthquake evacuation preparedness in buildings. Advanced Engineering Informatics, 45, 101118.

17. Fujimi, T., \& Fujimura, K. (2020). Testing public interventions for flash flood evacuation through environmental and social cues: The merit of virtual reality experiments. International Journal of Disaster Risk Reduction, 101690.

18. Gil, Y., David, C. H., Demir, I., Essawy, B. T., Fulweiler, R. W., Goodall, J. L., J.L., Karlstrom, L., Lee, H., Mills, H.J., Oh, J.H., Pierce, S. A. (2016). Toward the Geoscience Paper of the Future: Best practices for documenting and sharing research from data to software to provenance. Earth and Space Science, 3(10), 388-415.

19. Han, Y., Liu, H., Tian, Y., Chen, Z., \& Nie, Z. (2018, November). Virtual reality oriented modeling and simulation of water-dropping from helicopter. In Proceedings of the 2018 International Conference on Artificial Intelligence and Virtual Reality (pp. 24-29).

20. Havenith, H. B., Cerfontaine, P., \& Mreyen, A. S. (2019). How virtual reality can help visualise and assess geohazards. International Journal of Digital Earth, 12(2), 173-189. 
21. Jadidoleslam, N., Mantilla, R., Krajewski, W. F., \& Goska, R. (2019). Investigating the role of antecedent SMAP satellite soil moisture, radar rainfall and MODIS vegetation on runoff production in an agricultural region. Journal of Hydrology, 579, 124210.

22. Kolb, D., Kurtz, W., Weismüller, J., Ramm, A. V., Ludwig, R., \& Kranzlmüller, D. (2019, January). Using virtual reality to visualize extreme rainfall events derived from climate simulations. In Geophysical Research Abstracts (Vol. 21).

23. Kruger, A., Krajewski, W.F., Niemeier, J.J., Ceynar, D.L. and Goska, R., 2016. Bridge-mounted river stage sensors (BMRSS). IEEE Access, 4, pp.8948-8966.

24. Labossière, L. M., \& McGee, T. K. (2017). Innovative wildfire mitigation by municipal governments: two case studies in Western Canada. International journal of disaster risk reduction, 22, 204-210.

25. Lankford, A. (2019). Confirmation That the United States Has Six Times Its Global Share of Public Mass Shooters, Courtesy of Lott and Moody's Data. Econ Journal Watch, 16(1), 69.

26. Leder, J., Horlitz, T., Puschmann, P., Wittstock, V., \& Schütz, A. (2019). Comparing immersive virtual reality and powerpoint as methods for delivering safety training: Impacts on risk perception, learning, and decision making. Safety science, 111, 271-286.

27. Li, X., Lv, Z., Wang, W., Zhang, B., Hu, J., Yin, L., \& Feng, S. (2016). WebVRGIS based traffic analysis and visualization system. Advances in Engineering Software, 93, 1-8.

28. Lv, Z. (2019). Virtual reality in the context of Internet of Things. Neural Computing and Applications, 1-10.

29. Lv, Z., Yin, T., Zhang, X., Song, H., \& Chen, G. (2016). Virtual reality smart city based on WebVRGIS. IEEE Internet of Things Journal, 3(6), 1015-1024.

30. Macchione, F., Costabile, P., Costanzo, C., \& De Santis, R. (2019). Moving to 3-D flood hazard maps for enhancing risk communication. Environmental Modelling \& Software, 111, 510-522.

31. Masrur, A., Zhao, J., Wallgrün, J. O., LaFemina, P., \& Klippel, A. (2017, October). Immersive applications for informal and interactive learning for earth science. In Proceedings of the Workshop on Immersive Analytics, Exploring Future Interaction and Visualization Technologies for Data Analytics (pp. 1-5).

32. MindCommerce. (2020). Virtual Personal Assistants (VPA) and Smart Speaker Market: Artificial Intelligence Enabled Smart Advisors, Intelligent Agents, and VPA Devices 2020 - 2025. Retrieved from https://mindcommerce.com/reports/virtual-personal-assistant-market/ 
33. Nahavandi, S., Wei, L., Mullins, J., Fielding, M., Deshpande, S., Watson, M., ... \& Jones, R. (2019, July). Haptically-enabled vr-based immersive fire fighting training simulator. In Intelligent Computing-Proceedings of the Computing Conference (pp. 11-21). Springer, Cham.

34. O'Rourke, M. (2017). How to Respond to an Active Shooter. Risk Management, 64(10), 40-41.

35. Pimont, F., Parsons, R., Rigolot, E., de Coligny, F., Dupuy, J. L., Dreyfus, P., \& Linn, R. R. (2016). Modeling fuels and fire effects in 3D: model description and applications. Environmental Modelling \& Software, $80,225-244$.

36. Salas, D., Liang, X., Navarro, M., Liang, Y., \& Luna, D. (2020). An open-data open-model framework for hydrological models' integration, evaluation and application. Environmental Modelling \& Software, 126, 104622.

37. Sermet, Y. and Demir, I., 2019a. Flood action VR: a virtual reality framework for disaster awareness and emergency response training. In ACM SIGGRAPH 2019 Posters (pp. 1-2).

38. Sermet, Y. and Demir, I., 2019b. Towards an information centric flood ontology for information management and communication. Earth Science Informatics, 12(4), pp.541-551.

39. Sermet, Y., \& Demir, I. (2018). An intelligent system on knowledge generation and communication about flooding. Environmental modelling \& software, 108, 51-60.

40. Sermet, Y., \& Demir, I. (2020). Virtual and augmented reality applications for environmental science education and training. New Perspectives on Virtual and Augmented Reality: Finding New Ways to Teach in a Transformed Learning Environment.

41. Sermet, Y., Demir, I., \& Muste, M. (2020a). A serious gaming framework for decision support on hydrological hazards. Science of The Total Environment, 138895.

42. Sermet, Y., Villanueva, P., Sit, M. A., \& Demir, I. (2020b). Crowdsourced approaches for stage measurements at ungauged locations using smartphones. Hydrological Sciences Journal, 65(5), 813822.

43. Simpson, M., Wallgrün, J. O., Klippel, A., Yang, L., Garner, G., Keller, K., ... \& Bansal, S. (2016). Immersive analytics for multi-objective dynamic integrated climate-economy (DICE) models. In Proceedings of the 2016 ACM companion on interactive surfaces and spaces (pp. 99-105).

44. Sit, M., Sermet, Y. and Demir, I., 2019. Optimized watershed delineation library for server-side and client-side web applications. Open Geospatial Data, Software and Standards, 4(1), p.8.

45. Stock, C., Bishop, I. D., O'Connor, A. N., Chen, T., Pettit, C. J., \& Aurambout, J. P. (2008). SIEVE: Collaborative decision-making in an immersive online environment. Cartography and Geographic Information Science, 35(2), 133-144. 
46. Sun, T., Li, B. C., \& Mao, B. (2019). Exploring Drivers' Behavior on the Highway in a Virtual Reality Environment. In CICTP 2019 (pp. 696-701).

47. Turner, C. J., Hutabarat, W., Oyekan, J., \& Tiwari, A. (2016). Discrete event simulation and virtual reality use in industry: New opportunities and future trends. IEEE Transactions on Human-Machine Systems, 46(6), 882-894.

48. U.S. Army. (2020, July 14). Army looks to better attract gaming industry for training simulations. Retrieved on July 28, 2020, from https://www.army.mil/article/237223/army looks to better attract gaming industry for training simulations

49. Visser, A. G., Beevers, L., \& Patidar, S. (2019). A coupled modelling framework to assess the hydroecological impact of climate change. Environmental Modelling \& Software, 114, 12-28.

50. Wallgrün, J. O., Masrur, A., Zhao, J., Taylor, A., Knapp, E., Chang, J. S. K., \& Klippel, A. (2019, March). Low-cost VR applications to experience real word places anytime, anywhere, and with anyone. In 2019 IEEE 5th Workshop on Everyday Virtual Reality (WEVR) (pp. 1-6). IEEE.

51. Wang, J., Chen, M., Lü, G., Yue, S., Wen, Y., Lan, Z., \& Zhang, S. (2020). A data sharing method in the open web environment: Data sharing in hydrology. Journal of Hydrology, 124973.

52. Wang, V., Salim, F., \& Moskovits, P. (2013). The definitive guide to HTML5 WebSocket (Vol. 1). New York: Apress.

53. Wang, W., Wu, X., He, A., \& Chen, Z. (2019). Modelling and Visualizing Holographic 3D Geographical Scenes with Timely Data Based on the HoloLens. ISPRS International Journal of GeoInformation, 8(12), 539.

54. Weber, L.J., Muste, M., Bradley, A.A., Amado, A.A., Demir, I., Drake, C.W., Krajewski, W.F., Loeser, T.J., Politano, M.S., Shea, B.R. and Thomas, N.W., 2018. The lowa Watersheds Project: lowa's prototype for engaging communities and professionals in watershed hazard mitigation. International journal of river basin management, 16(3), pp.315-328.

55. Wikipedia contributors. (2020, June 22). List of mass shootings in the United States in 2019. In Wikipedia, The Free Encyclopedia. Retrieved 21:44, July 12, 2020, from https://en.wikipedia.org/w/index.php?title=List of mass shootings in the United States in $\underline{2019}$

56. Winkler, D., Zischg, J., \& Rauch, W. (2018). Virtual reality in urban water management: communicating urban flooding with particle-based CFD simulations. Water Science and Technology, 77(2), 518-524. 
57. Xie, C. Y., Luo, J. R., \& Guo, L. L. (2011). Probabilistic analysis of radioactive materials release arising from transportation accident. Journal of Safety and Environment, 11(4), 242-244.

58. Xu, H., Windsor, M., Muste, M. and Demir, I., 2020. A web-based decision support system for collaborative mitigation of multiple water-related hazards using serious gaming. Journal of Environmental Management, 255, p.109887.

59. Yildirim, E., \& Demir, I. (2019). An integrated web framework for HAZUS-MH flood loss estimation analysis. Natural Hazards, 99(1), 275-286.

60. Zhang, F., Chen, M., Ames, D.P., Shen, C., Yue, S., Wen, Y. and Lü, G., 2019. Design and development of a service-oriented wrapper system for sharing and reusing distributed geoanalysis models on the web. Environmental Modelling \& Software, 111, pp.498-509.

61. Zhu, R., Becerik-Gerber, B., Lucas, G., Southers, E., \& Pynadath, D. V. (2019). Information Requirements for Virtual Environments to Study Human-Building Interactions during Active Shooter Incidents. In Computing in Civil Engineering 2019: Smart Cities, Sustainability, and Resilience (pp. 188-195). Reston, VA: American Society of Civil Engineers. 